\title{
The Undergraduate Scientific and Technological Innovation and Entrepreneurship Education Platform
}

\author{
Jin Bao Song ${ }^{1, a}$ \\ ${ }^{1}$ Information Engineering School, Communication University of China, Beijing, China
}

\begin{abstract}
Along with the development of innovation and entrepreneurship nowadays, how to promote the undergraduate scientific and technological innovation and entrepreneurship during the period of university education is more and more discussed by the people. How to organize and manage effectively for the undergraduate scientific and technological innovation and entrepreneurship, how to give the undergraduate scientific and technological innovation and entrepreneurship some pointed guide and cultivation, how to form an organic quality training mechanism together with class education and social education are important problems which effect undergraduate scientific and technological innovation and entrepreneurship. Firstly, the undergraduate scientific and technological innovation and entrepreneurship service platform is introduced in this paper to solve these above problems. Secondly, the new mode of scientific and technological innovation and entrepreneurship ability training for the undergraduates is analyzed. At last, a new way of thinking for the scientific and technological innovation and entrepreneurship service is provided the talent training in this paper which will play an important role on the development of innovation and entrepreneurship education.
\end{abstract}

\section{Introduction}

The undergraduate ability of scientific and technological innovation and entrepreneurship is one of the important contents of China's higher education talent training. It is also an important educational factor to improve the comprehensive quality of the undergraduates. It plays an important role on the cultivation of the students' competence, cooperation ability, even the leadership and the ability to make decisions. And it is the important means to meet the social demand and promote the undergraduate employment. Constructing the scientific and technological innovation country and the scientific and technological and innovative city has become a national and Beijing's important planning construction goal [1]. The Communication University of China is a famous university in the field of information transmission in China, which has outstanding contribution and the important position in media content and form innovation research, media high technology research and development and the unit decision consultation service for the enterprise or business and so on. It is an important source of the media science and technology innovation. A lot of the media realm student's innovation achievements become productive forces to promote the scientific and technological achievements through guide and cultivation, which will greatly promote the economic growth of the country [2].

\footnotetext{
a Corresponding author: songjinbao@cuc.edu.cn
}

\section{The significance of undergraduate science and technology innovation and entrepreneurship service platform}

The construction of undergraduate science and technology innovation and entrepreneurship service platform is based on the science and technology innovation park of the Communication University of China, which provides service to the full-time undergraduate and graduate students. This platform is an important part of the professional incubation service project in the modern service industry. This platform plays an important role in driving the whole environmental construction of the country's science and technology.

How to organize and manage effectively for the undergraduate scientific and technological innovation and entrepreneurship, how to give the undergraduate scientific and technological innovation and entrepreneurship some pointed guide and cultivation, how to form an organic quality training mechanism together with class education and social education are important problems which effect undergraduate scientific and technological innovation and entrepreneurship. And we must put forward the effective solution. The undergraduate science and technology innovation and entrepreneurship services platform construction is to solve these problems. The core of the platform is the 
innovation and entrepreneurship quality ability training. The goal of the platform is to promote science and technology innovation incubation and achievements of the media. The platform provides effective information management and service, innovation and entrepreneurship training and guidance, coordination and mutual communication platform for college students with the aid of new media network, modern means of science and technology. The construction and implementation of undergraduate science and technology innovation and entrepreneurship service platform has important significance to improve enthusiasm of undergraduate innovation and enterprise, promote the produce and accelerate conversion of the university students' scientific and technological achievements, improve the success rate of entrepreneurship, regulate the college students' innovative and enterprising organization and management and form the innovation and interactive environment including university students, experts, enterprises and departments of government.

\section{The construction goal of undergraduate science and technology innovation and entrepreneurship service platform}

The science and technology innovation and entrepreneurship service platform is to support undergraduate innovative and enterprising work as the core target and put public welfare, demonstration, profession as the main characteristics. The specific goals are as follow:

1) The $B / S$ interactive network is as the foundation platform, combined with the new media, virtualization and other modern means of science and technology [3]. It is for the technical integration and demonstration of information management and service, innovation and entrepreneurship training and guidance, coordination and mutual communication platform construction around undergraduate science and technology innovation and entrepreneurship activities. The Communication University of China undergraduate science and technology innovation activities comprehensive service demonstration system is established including organization, management, training, promotion, resources sharing, the information communication. And the employment and entrepreneurship comprehensive service demonstration system of Communication University of China science and technology park is also set up including entrepreneurship dynamic and hotspots tracking, employment guidance, business training, achievement presentation and investment hatch, project tracking and coordination and communication.

2) The establishment of Communication University of China undergraduate science and technology innovation and entrepreneurship service platform software and hardware test environment and operating system environment will be completed, including undergraduate science and technology innovation and entrepreneurship information service platform internal network, central server, video server, cooperation and sharing resources server, data information server, user authentication server and browser based client software and security plug-ins, etc [4].

3) Based on the platform, for the Communication University of China and its science and technology park, a group of outstanding entrepreneurs of college students are cultivated. A coaching professional team is formed. A group of college students' successful entrepreneurship models are set up. All kinds of relevant information of the enterprises and projects for college students are provided. The business consulting and training are organized in order to improve all kinds of the entrepreneurs' skills. And enterprise fast growth and independent business are promoted [5].

4) Based on the platform, the university science park industry chain is effectively extended. The new pattern of "complementary advantages and linkage development" is gradually formed with other industries inside and outside the park. The multiplication effect of entrepreneurship is realized in order to create more employment opportunities, ease Beijing and radiation regional employment pressure and promote the regional economic growth.

The Communication University of China undergraduate science and technology innovation and entrepreneurship service platform is divided into two parts:

1) Communication University of China science and technology park undergraduate science and technology innovation activities comprehensive service system:

a) A media organization management system of science and technology innovation team is established, including team building system, the daily operation management system, the reward system, coordination mechanism. The objectives and tasks of the innovative team are specified.

b) The online training and technical guidance environment are provided, focused on training management of the service around university students media science and technology training and innovation technical guidance.

c) The project results display platform is established for media science and technology innovation team project intermediary and display needs. The innovation and organization management platform is provided for the media project application and competition of national and municipal science and technology innovation.

d) The equipment and experimental environment information management platform is established in order to provide the necessary experiment and development conditions for media science and technology innovation team, including equipment and laboratory management, status enquiry, appointment management and necessary training.

e) A scientific and technological innovation BBS and media team communication space are established. The teams BBS plate and communication space are built according to professional direction. The online interactive innovation environment is formed.

2) Communication University of China science and technology park undergraduate entrepreneurship comprehensive service system: 
a) The interactive college students' employment information release and online recruitment platform is established in order to provide bridge for college students' employment and the enterprises and recruitment direct to innovative team and the outstanding students.

b)The entrepreneurship policy guidance, business dynamic and hot tracking, entrepreneurship case analysis, online entrepreneurship training, science and technology resources service, innovation service navigation and so on are provided focusing on career guidance and training.

c) The results show and hatch investment link is provided and the hatch and entrepreneurial tracking information management mechanism is set up according to the needs of the entrepreneurship development.

d) The online collaboration and mutual communication platform is created, implementing online meeting and cooperation project development, white board data synchronization, audio interaction, the resource exchange and sharing function.

e) A media technology entrepreneurship BBS is established [6].

\section{The overview of undergraduate science and technology innovation and entrepreneurship service platform}

The Communication University of China undergraduate science and technology innovation and entrepreneurship service platform is divided into two systems. One is undergraduate science and technology innovation activities comprehensive service system. The other is undergraduate entrepreneurship comprehensive service system.

\subsection{Science and technology innovation service system}

The software system frame of science and technology innovation service system is shown as Figure 1. This system is mainly divided into six big modules including user login module, the project application and management module, the innovation achievements exhibit module, online training and technical guidance module, equipment and the experiment environment module and team communication BBS module. The modules specific functions are shown below.

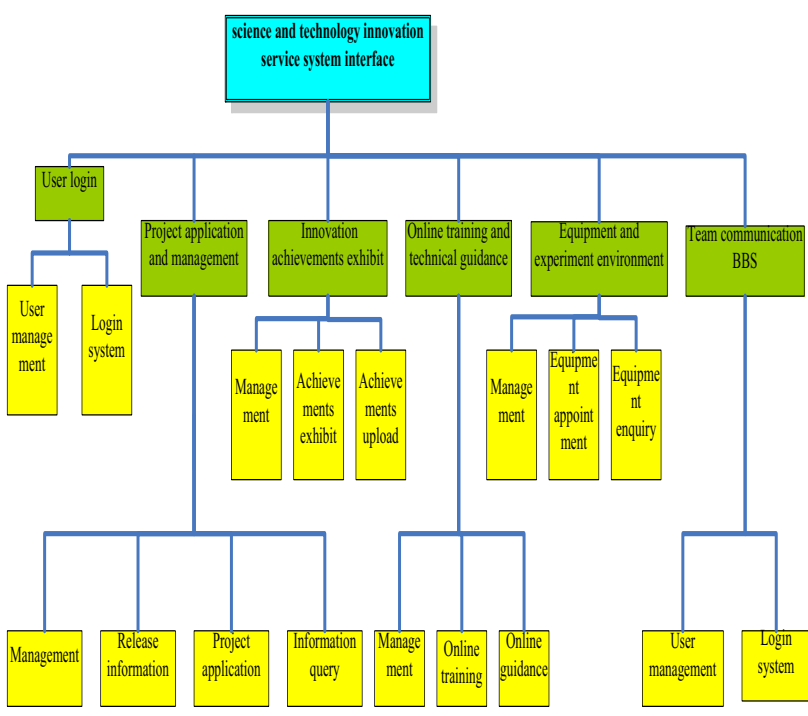

Figure 1. Science and technology innovation service system diagram

\subsection{Entrepreneurship service system}

The software system frame of entrepreneurship service system is shown as Figure 2. This system is mainly divided into six big modules including user login module, employment information and online recruitment module, training and policy guidance module, business incubation and entrepreneurial track module, the online collaboration and mutual communication module, team communication BBS module. Among them except employment information and online recruitment module and online collaboration and mutual communication outside module, other modules are similar to innovation service system corresponding modules. The modules specific functions are shown below.

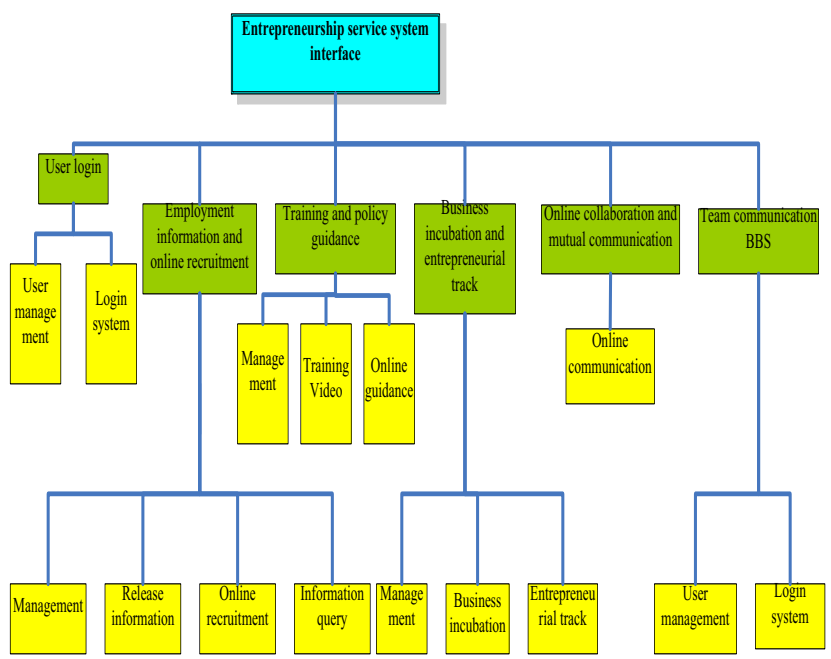

Figure 2. Entrepreneurship service system diagram

\section{Development environment}

\subsection{Software environment summary}


- $\quad$ server system: Windows server 2003

- database: mysql 5.1 above

- database graphic interface: navicat more than 9.0

- IDE: myeclipse 8.6 and above

- development of the main language: Java

- JDK version: JDK 1.6 above

- Java web containers: tomcat 6.0 above

- development framework: SSH

- the browser: firefox or chrome

\subsection{Software environment introduction}

(1) JDK:

Java SE6 software development kit, is a Java applications foundation. The J2EE is based on Java technology, so installation JDK must be configuration of J2EE environment.

(2)Tomcat:

Tomcat is a JSP engine developed by Apache organization. It has the function of the Web server, and could be used as independent Web server. But, as a Web server, Tomcat doesn't process static HTML pages more quickly and strongly than Apache. So the Apache and Tomcat is used together by letting the Apache provides processing for the Web static page services and Tomcat JSP engine provides JSP analysis in order to get better performance. Tomcat itself is a sub-project Apache, so that Tomcat provides powerful support to Apache.

(3)MySQL:

MySQL database system uses the most commonly used database management language-structured query language (SQL) for database management. Using the database graphical interface Navicat can directly add data.

(4)MyEclipse:

MyEclipse is a free IDE integrated development environment tool which supports the development of language and installing plugins can make its function more powerful.

\subsection{Struts 2, Spring, Hibernate}

$\mathrm{SSH}$ is the abbreviation of Struts2, Spring, Hibernate. It is a very popular Java Web development framework in the current Java world. Also it is a kind of the MVC. MVC is Model, View, Controller layered structure. The three parts should as far as possibly reduce coupling to improve application expansibility and maintainability, shown as Figure 3. Struts 2 is mainly as a control layer, shown as Figure 4. Spring is mainly used for business layer, shown as Figure 5. Hibernate is database lasting layer, shown as Figure 6.

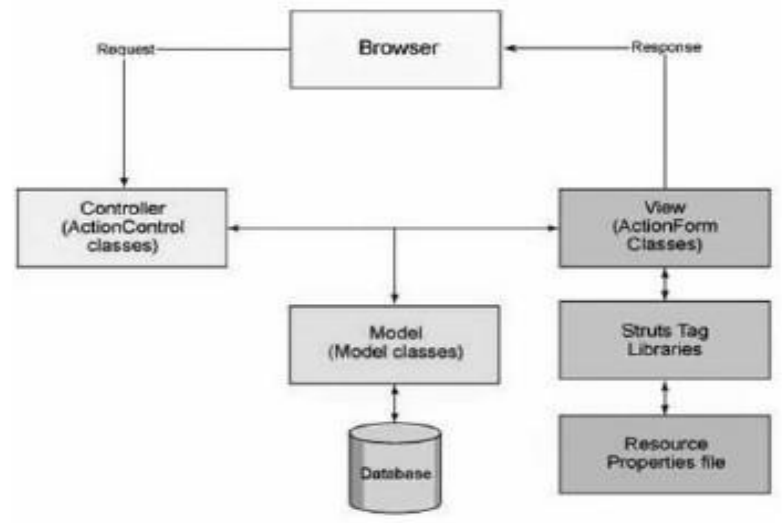

Figure 3. MVC schematic framework

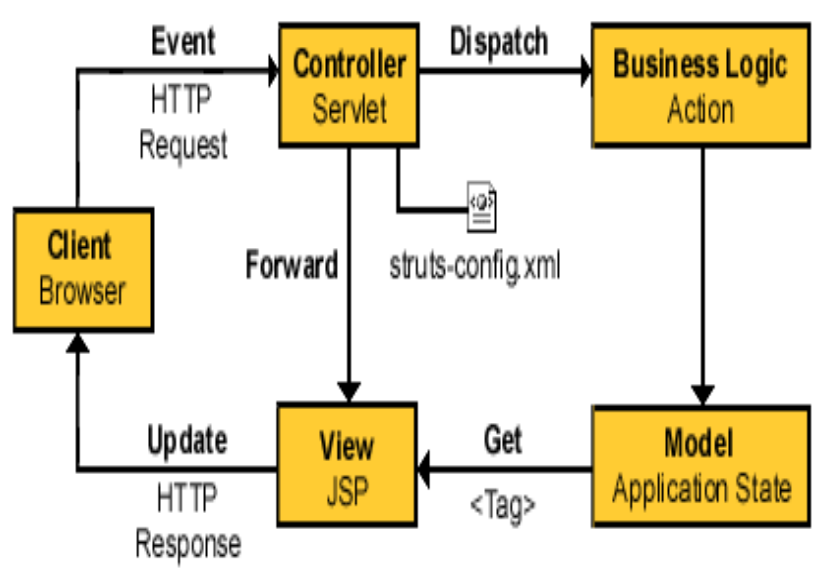

Figure 4. Structs schematic framework
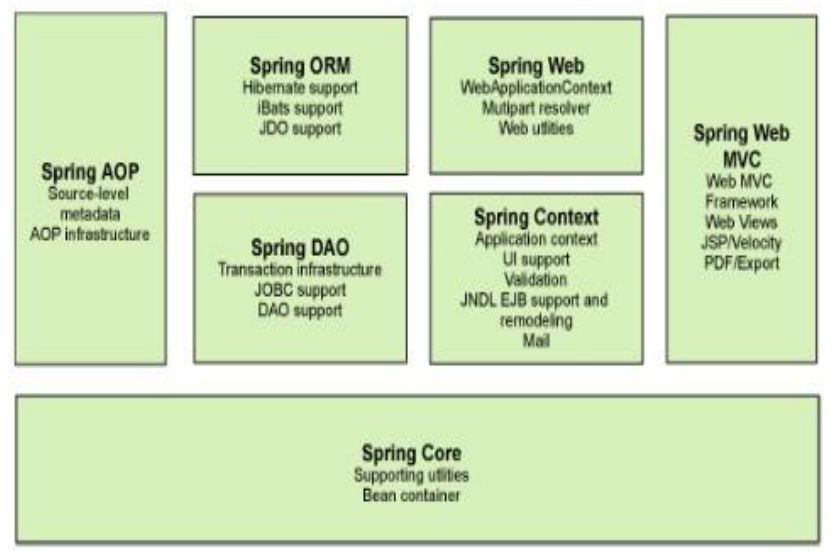

Figure 5. Spring schematic framework 


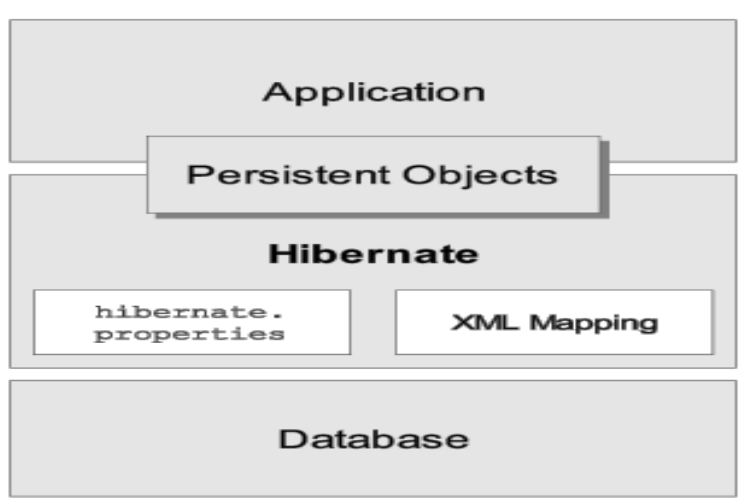

Figure 6. Hibernate schematic framework

\section{Summary}

In the undergraduate science and technology innovation and entrepreneurship services platform construction, we use the technical route of "architecture design" $\rightarrow$ "technical model research" $\rightarrow$ "prototype system construction" $\rightarrow$ "demonstration application and perfect system ".

1) We have deeply researched the general framework of undergraduate innovation and business information service platform. In view of the specific requirements of the college students' extracurricular scientific research and science and engineering practice activities, employment, business aspects, and organizational management, innovative and undertaking training, policy guidance, project investment promotion, hatch, equipment resources sharing, online meeting and cooperation, we have done further discussion and demand function analysis, which determines the undergraduate science and technology innovation and entrepreneurship information service platform constraint condition and basic mode.

2) According to the demand analysis and the overall framework of undergraduate science and technology innovation and entrepreneurship information services platform, we have designed the internal structure of the system and researched the Ajax, XMPP, MVC technology[7-9], established technical model that meets demand and architecture requirement, realized the relevant function module, and at the same time synchronously carried on technological verification to ensure the feasibility, reliability and scalability of the technology and the business standard.

3) We have further integrated the software and hardware resources of the undergraduate science and technology innovation and entrepreneurship information service platform, finished a practical integration development, designed and established undergraduate extracurricular technological innovation activities information service demonstration system and undergraduate employment venture information service demonstration system prototype and integrated and tested system to test system function, performance requirements.

4) We have established a practical application of the hardware and software environment. Through actual application verification and testing system at the park of science and innovation and related universities, we have gotten through technology difficult points, realized the fusion penetration and general test of the technology, and verified the feasibility and reliability of the overall scheme. Also, according to validation and evaluating the effect of the system, we have adjusted and optimized system solutions and perfected the system platform.

\section{References}

1. J. B. Song. (2012, June). The Communication University of China undergraduate science and technology innovation and entrepreneurship services platform research report. Communication University of China, Beijing, China.

2. H. Y. Ma. (2012, June). The Communication University of China undergraduate science and technology innovation and entrepreneurship services platform work report. Communication University of China, Beijing, China.

3. Z. B. Liu, Java WEB core framework. Beijing: Posts \& Telecom Press, 2008, pp. 123-135.

4. W. Q. Sun, Proficient Hibernate JAVA object persistent mass media technology, Beijing: Posts \& Telecom Press, 2007, pp. 212-236.

5. R. Johnson, J. Hoeller, Expert one-on-one J2EE Development without EJB, 2005.

6. C. Bauer, G. King, JAVA Persistence with hibernate, 2008.

7. J. Sack, sql server 2008 transact-sql recipes, 2010.

8. R. Stephens, Beginning database design solutions, 2010

9. M. Fowler, Patterns of Enterprise Application Architecture, 2011. 